\title{
Experiences with a voluntary surveillance system for early detection of equine diseases in Switzerland
}

\author{
R. STRUCHEN ${ }^{1 *}$, D. HADORN ${ }^{2}$, F. WOHLFENDER ${ }^{1,3}$, S. BALMER $^{2}$, \\ S. SÜPTITZ ${ }^{2}$, J. ZINSSTAG ${ }^{4}$ AND F. VIAL ${ }^{1}$ \\ ${ }^{1}$ Veterinary Public Health Institute, Vetsuisse Faculty, University of Bern, Switzerland \\ ${ }^{2}$ Swiss Federal Food Safety and Veterinary Office, Bern, Switzerland \\ ${ }^{3}$ Institut suisse de médecine équine, Vetsuisse Faculty, University of Bern, Switzerland \\ ${ }^{4}$ Swiss Tropical and Public Health Institute, University of Basel, Switzerland
}

Received 7 April 2015; Final revision 22 December 2015; Accepted 8 January 2016; first published online 5 February 2016

\section{SUMMARY}

Clinical observations made by practitioners and reported using web- and mobile-based technologies may benefit disease surveillance by improving the timeliness of outbreak detection. Equinella is a voluntary electronic reporting and information system established for the early detection of infectious equine diseases in Switzerland. Sentinel veterinary practitioners have been able to report cases of non-notifiable diseases and clinical symptoms to an internet-based platform since November 2013. Telephone interviews were carried out during the first year to understand the motivating and constraining factors affecting voluntary reporting and the use of mobile devices in a sentinel network. We found that non-monetary incentives attract sentinel practitioners; however, insufficient understanding of the reporting system and of its relevance, as well as concerns over the electronic dissemination of health data were identified as potential challenges to sustainable reporting. Many practitioners are not yet aware of the advantages of mobile-based surveillance and may require some time to become accustomed to novel reporting methods. Finally, our study highlights the need for continued information feedback loops within voluntary sentinel networks.

Key words: Infectious diseases, mobile devices, sentinel networks, veterinary practitioners, voluntary reporting.

\section{INTRODUCTION}

Widespread access to the internet and mobile phones over the past decade has promoted the use of modern communication technologies to collect human and animal health data [1-3]; while new approaches to outbreak detection, such as syndromic surveillance, have simultaneously emerged to further strengthen human and animal health surveillance [4].

\footnotetext{
* Author for correspondence: Ms. R. Struchen, Veterinary Public Health Institute, Schwarzenburgstrasse 155, 3003 Bern, Switzerland. (Email: rahel.struchen@vetsuisse.unibe.ch)
}

Mobile phone applications can benefit disease surveillance by increasing speed and automation of data collection, providing accurate geo-location data, and allowing for rapid two-way transfer of information between data collectors and data users/ analysts [5]. While the development of mobilephone-based participatory systems for human public health has really taken off [6], they are yet to be extensively exploited for animal disease surveillance. Mobile phone technologies can be particularly beneficial to large-animal veterinarians who, in contrast to general practitioners or companion-animal veterinarians, visit their patients on their premises. 
Reliable surveillance systems are needed to reduce the impacts of emerging, and potentially zoonotic, diseases on animal and human health and the primary sector. Consequently, the willingness of veterinarians to continuously report their observations is essential for the successful implementation of practitionerbased surveillance systems [7]. However, little is known about previous experiences in implementing mobile technologies for veterinary diseases surveillance systems $[2,3,8]$; or about the factors that motivate or constrain veterinary practitioners to submit clinical data to surveillance programmes, although a few studies looked into these factors in the context of laboratory-based surveillance systems [9-11].

In Switzerland, a new electronic reporting and information system for the monitoring of equine health, Equinella, has been operational since November 2013. We present here results from its first year during which we aimed to better understand the motivations and barriers to voluntary participation in practitionerbased surveillance systems; and evaluated the suitability of mobile devices to collect animal health data in a timely fashion for surveillance.

\section{EQUINELLA SYSTEM}

Equinella was first established in 1990 and primarily focused on the paper-based reporting of nonnotifiable $\uparrow$ equine diseases by a network of sentinel veterinary practitioners. In 2012, an evaluation of the system showed that it was no longer representative of the Swiss horse population [12] (with only six reports received in 2012); and a survey performed among veterinarians revealed that most veterinarians would prefer to report cases electronically; and that many were willing to additionally report syndromes in addition to disease cases [13].

The new Equinella was re-launched in 2013 as a collaborative surveillance system run by the Federal Food Safety and Veterinary Office (FSVO), the Vetsuisse Faculty (Bern) and the Swiss Association of Equine Practitioners. It relies on the reporting of cases of non-notifiable diseases as well as clinical symptoms through any device with an internet connection and a web browser installed such as desktop computers or mobile devices like smartphones or tablets.

\footnotetext{
$\dagger$ Notifiable diseases according to the Swiss Animal Health Ordinance (AHO, SR 916.401) must be reported to the cantonal veterinary office by telephone.
}

To guarantee data protection and facilitate data management, sentinel practitioners must register. Sentinel veterinarians were recruited in September 2013 from both mixed-animal and purely equine practices through mailing lists and an article published in the journal of the Society of Swiss Veterinarians [14]. Participating practitioners do not receive monetary compensation but benefit from various nonmonetary incentives. These include password-secured access to an interactive overview of all incoming, anonymized reports; a monthly electronic newsletter relaying national and international equine health news; and a mobile phone text message service to alert them in case of an outbreak. They can contact and draw from the expertise of the Equinella support team and attend one free professional development course per year. Additionally, smartphones specialized for outdoor use (ESP, see Supplementary material) were provided to those indicating an interest in using such a device for reporting directly from the field.

To facilitate and standardize data collection, veterinarians can choose clinical symptoms or diseases (Supplementary material) from pre-defined check lists. Data on the equid and the holding visited are collected. The ID of the reporting veterinarian and the date of the report are automatically recorded by the system. Additional information can be entered in a free text box and pictures can be uploaded. A reminder email is automatically sent to all sentinel veterinarians once a month. Recipients can then either confirm that they had no clinical observations of relevance to Equinella in their practice in the previous month or they can report their observations retrospectively.

\section{PARTICIPATION}

The number of sentinel veterinarians increased from 39 in December 2013 to 67 in November 2014 (Fig. 1), with only one practitioner dropping out. Two continuing professional development courses were organized in October and November 2014. The possibility for registered practitioners to attend one of them for free together with the increased publicity around Equinella at that time probably explain the increased number of registrations during these 2 months.

A relatively high participation was found throughout the first year [median $73 \%$, interquartile range (IQR) 70-76, Fig. 1] computed as the monthly proportion of sentinel veterinarians that either submitted 


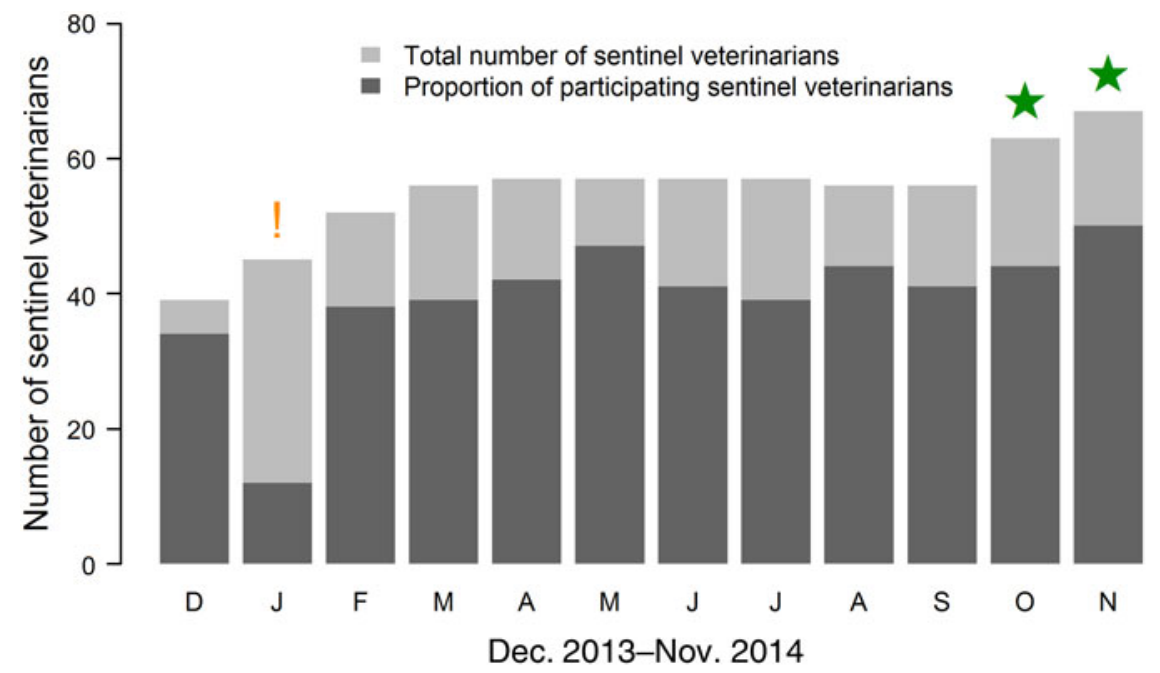

Fig. 1. Participation of the sentinel veterinarians to the new Equinella system within its first operational year. Light grey bars represent the monthly number of registered sentinel veterinarians. Dark grey bars represent the monthly number of participating sentinel veterinarians (i.e. that either submitted a report or confirmed not having observed any relevant cases in their practice area during the preceding month). The orange exclamation mark (!) indicates a technical problem which prevented many sentinel veterinarians from confirming that they did not have observations to report. The green stars $(\star)$ indicate the months when continuing professional development courses were organized.

a report or confirmed not having observed any relevant cases. The drop (27\%) in January 2014 was caused by a technical problem with the reminder e-mails, resulting in many sentinel veterinarians not being able to confirm that they did not have observations to report. A comparable participation was found at the practitioner-level (proportion of months a sentinel veterinarian participated: median 75\%, IQR 54-2-91·1).

Between December 2013 and November 2014, a total of 78 reports were submitted to Equinella by 24 sentinel veterinarians: 35 reports $(44.9 \%)$ included only clinical symptoms, six (7.7\%) only diseases and $37(47 \cdot 4 \%)$ had both. The median number of reports received per month was six (IQR 3.8-9). These numbers constitute a real improvement from the reporting frequency observed in the last years of the paper-based surveillance system. Mobile devices were infrequently used for reporting with only 10 reports $(12 \cdot 8 \%)$ submitted using mobile devices (none of which were ESP) by five different sentinel veterinarians. A minor, but non-significant improvement (Wilcoxon rank sum test $422 \cdot 5, P=0 \cdot 215$ ) in reporting timeliness (difference in days between the diagnosis date and the reporting date) was found when using mobile devices (median 5.5 days, IQR $0-8.5$ ) over desktop computers (median 6.5 days, IQR 0-16.3). Although no precise data on reporting timeliness are available for the old paper-based Equinella, prior to 2013, participants were encouraged to mail forms to the Federal
Veterinary Office (now FSVO) every 2 weeks [15]. The timeliness of the new electronic system is therefore most likely a significant improvement on its predecessor. Still, our findings were surprising as $45 \%$ of the veterinarians surveyed in 2012 said they would be willing to report on a daily basis when cases were observed [13]. While, in an ideal surveillance system, cases would be reported to the authorities on the day they were observed, a reporting timeliness of less than a week remains acceptable for the early detection purpose of the system and is comparable to the reporting delay observed in other animal health data sources $[16,17]$.

\section{INTERVIEWS}

Telephone interviews were conducted in May-June 2014 $(n=6)$, investigating the reasons for the initial low reporting frequency, and in November $2014(n=5)$, focusing on the use of mobile devices. Interviews were based on a series of primarily open questions and protocols were generated for all interviews (Supplementary material). For both sets of interviews, a convenience sample of registered veterinarians was used, with the second drawn only from those having an ESP.

\section{Motivation}

Receiving up-to-date information on the health status of the population under surveillance was stated as a 
strong incentive for participation in Equinella. This was especially important for small practices without close contacts to the university referral clinics or the larger clinics. The interviews highlighted the value of practitioners knowing that certain diseases still occur in Switzerland or that there are clinical manifestations present which are not observed in their own practice. Some interviewees mentioned that receiving such information made them feel better prepared to alleviate their customers' concerns and answer their questions. Further benefits included (1) an improved flow of information between veterinarians; (2) knowing what to expect in potentially risky situations (e.g. in auctions); and (3) reaching a correct diagnosis based on the knowledge of the current health situation. Being aware of the relevance of international animal movements for disease spread, one practitioner mentioned expanding the Equinella system to neighbouring countries. Another suggested that the treatments administered and their outcomes could be reported in the future.

However, many practitioners did not make full use of the Equinella information platform, often only accessing a single information source (such as the list of incoming reports, the interactive map or the latest newsletter). This may be the result of the time constraints of busy practitioners as well as their unfamiliarity with the electronic dissemination and display of health information.

\section{Reasons for low reporting frequency}

Approximately half of the interviewees believe that the low reporting frequency reflects the good health situation of the equine population in Switzerland, although some admitted to not consistently reporting new cases. The choice of an electronic reporting method was not unanimous among interviewees, one preferring a paper-based system and another expressing concerns over the dissemination of (potential erroneous or sensitive) information over new media, such as the internet.

The trade-off between user-friendliness and security needs was considered when designing the Equinella electronic system. Before a disease report is published online, an Equinella expert verifies each report and contacts the reporting sentinel veterinarian if anything is unclear. Despite all reports being anonymized before publication, communication between the Equinella team, the practitioners and their clients, regarding data privacy must be improved. A practitioner explained that some animal holders fear being considered as a risk to others, and therefore do not wish disease cases occurring on their holdings to be reported.

Interviewees who had not reported to Equinella stated that they had made no appropriate observations in their practice area - with a single exception in which the participant considered the reporting procedure as too complicated. However, the interviews delivered insight into how reports submitted to Equinella by sentinel veterinarians might be biased. The perceived low relevance of the system by some participants and an insufficient knowledge about the diseases and clinical symptoms that can be reported became apparent during the interviews. Two interviewees admitted never having read the list of diseases and clinical symptoms that had been actively communicated; while one interviewee had not yet submitted a report to Equinella because they had not dealt with cases they considered relevant. This sentiment was echoed in the words of another participant: 'For us practitioners, such a system is only relevant for infectious disease outbreaks and that mainly concerns equine herpes virus and maybe strangles. Regarding everything else, it is actually not in our interest to know what is happening in the neighbourhood.' Another interviewee said that they would only report a disease once confirmed by a laboratory test since a report of suspected disease without laboratory confirmation may easily be misinterpreted.

For a new surveillance system to be successfully implemented, it is essential that the sentinel practitioners fully understand the purpose and functionality of the system. Sustained communication between the data providers and the system users should facilitate a better understanding. In our case, improving and continuing our communication efforts, particularly on the topic of the clinical symptoms of interest for syndromic surveillance, may help to reduce some of the shortcomings identified in this pilot study.

\section{Reporting and use of mobile devices}

The uptake of mobile devices for reporting was low. Our suspicion that many practitioners were not fully aware of the advantages of mobile reporting was confirmed during the interviews. One practitioner felt mobile reporting to be unnecessary while another explained that they preferred reporting from the comfort of their home (irrespective of the device) seeing no benefit in doing so from the field. We found that the 


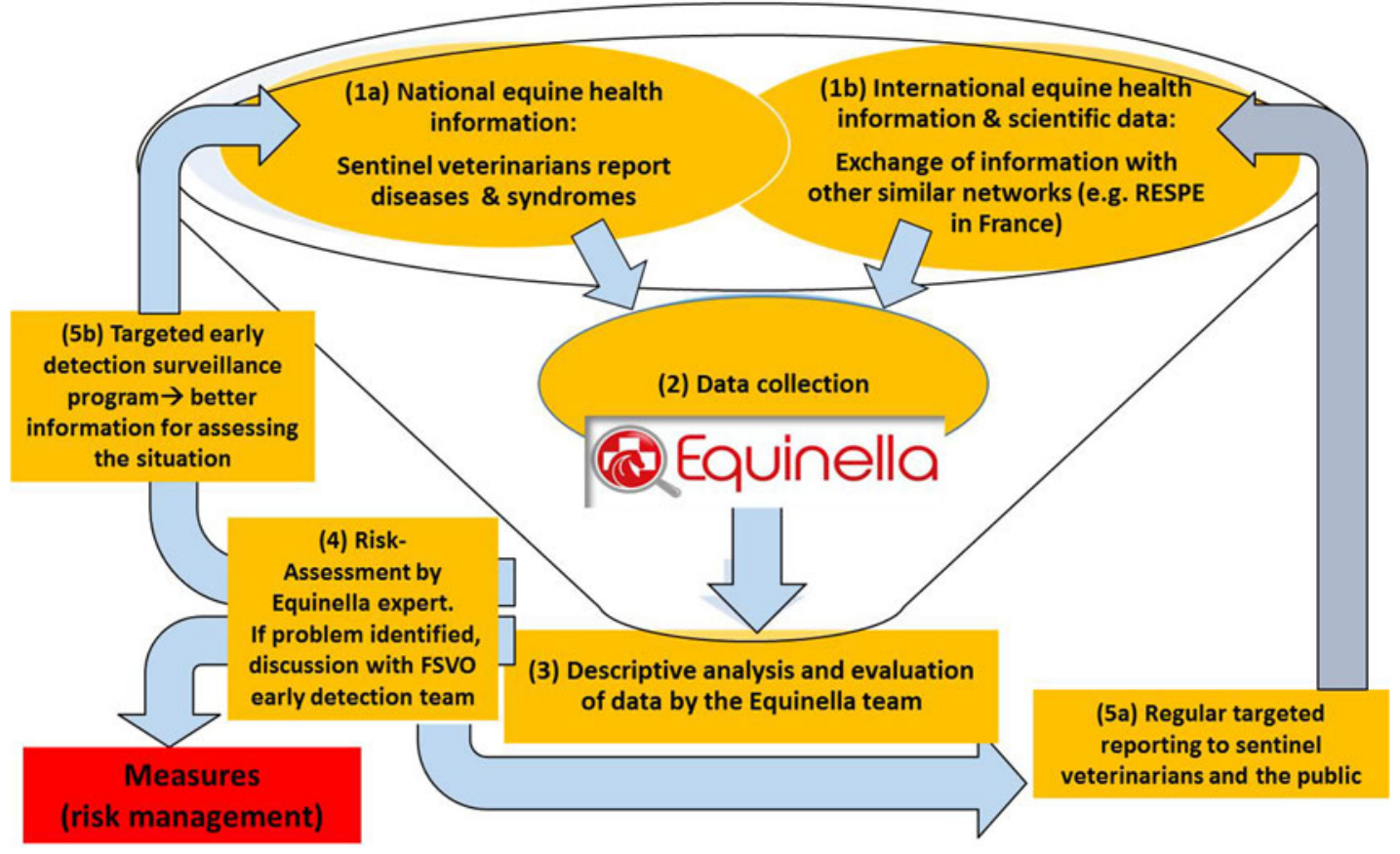

Fig. 2. Role of Equinella in providing information for effective veterinary public health action.

large-scale distribution of ESP phones, as an additional incentive to mobile reporting, to be challenging due to individual preferences regarding operating systems or mobile devices. Despite some sentinel veterinarians reporting having had positive experiences with the ESP in the field (due to its robustness), other ESP owners did not use the phones at all.

Several practitioners expressed a basic aversion to the use of mobile devices. It is therefore important, when designing new surveillance systems, to take into account the fact that the uptake of novel reporting methods may be slow as practitioners may require time and extra incentives to overcome possible technological aversions.

\section{CONCLUSIONS}

Our findings indicate that a voluntary surveillance system based on non-monetary incentives has the potential to attract sentinel practitioners. For example, offering regular relevant professional development courses may help recruit additional sentinel veterinarians in the future. Different types and formats of equine health information useful to practitioners were identified during the interviews. This will help the Equinella team to improve information feedback to data providers. Whether these incentives will be enough to keep motivation at a high level and ensure sustainable participation over the long-term still remains questionable, and participation needs to be monitored in the coming years. Understanding the barriers to participation might be further improved by interviewing veterinarians who have not yet registered.

Within the FSVO framework of establishing a national early detection system for emerging and reemerging diseases, Equinella represents a pilot system to evaluate how a network of sentinel veterinarians can contribute to the early detection of animal disease outbreaks. For example, reporting of non-notifiable diseases such as equine influenza or strangles is of relevance due to their considerable economic impacts on the horse industry sector [18, 19]. Spatio-temporal clusters of reported clinical symptoms such as fever of unknown origin, neurological or respiratory symptoms, or abortions might provide an early signal of the presence of a tropical or emerging disease such as West Nile virus [20] or equine herpes virus. The role of surveillance is to provide information for effective veterinary public health action (Fig. 2). All incoming reports are evaluated by an Equinella expert and interpreted in light of the national and international equine health situation and other available scientific data. If a potential problem is identified during this risk assessment phase, the early detection team at the FSVO is contacted to discuss potential measures. In the first 
12 months of Equinella, treatment schemes were jointly set up with veterinarians, sampling plans for further diagnostic analysis were proposed, and advice on hygiene or isolation measures to prevent further spread of the disease was given. A descriptive analysis of the reports is prepared and published via a newsletter and on the Equinella website to increase disease awareness and preparedness among practitioners.

We encountered some hurdles with the sporadic use of mobile devices and the lack of awareness of the advantages of mobile reporting during the first year. Our experiences in terms of overall participation were positive and many of the barriers to reporting we identified can be addressed in the future, making the outcome of the pilot project favourable. The use of a web-based application enabling individuals to report from their preferred device may increase the overall acceptability of the system and its sustainability. The Swiss veterinary authorities are now holding discussions to extend the concept to other animal species (e.g. cattle or pigs) which would also cover a broader spectrum of zoonoses of relevance to public health.

\section{SUPPLEMENTARY MATERIAL}

For supplementary material accompanying this paper visit http://dx.doi.org/10.1017/S0950268816000091.

\section{ACKNOWLEDGEMENTS}

The authors thank the 11 sentinel veterinarians who took part in the telephone interviews; all sentinel veterinarians for their valuable contribution to Equinella; the team at 4eyes for developing and implementing the electronic system; Ernest Peter for his contribution as technical project leader; Claudia Graubner for professional input and support; Patrick Presi for organizing the Equinella smartphones; Martin Reist and Andrew Tedder for helpful inputs; Marie-Eve Cousin for valuable support regarding the interviews; and the FSVO for funding this work.

\section{DECLARATION OF INTEREST}

None.

\section{REFERENCES}

1. Chunara R, Freifeld CC, Brownstein JS. New technologies for reporting real-time emergent infections. Parasitology 2012; 139: 1843-1851.
2. Madder M, et al. E-surveillance in animal health: use and evaluation of mobile tools. Parasitology 2012; 139: 1831-1842.

3. Walker J. New media methods for syndromic surveillance and disease modelling. CAB Reviews: Perspectives in Agriculture, Veterinary Science, Nutrition and Natural Resources 2013; 8: 1-13.

4. Rodríguez-Prieto V, et al. Systematic review of surveillance systems and methods for early detection of exotic, new and re-emerging diseases in animal populations. Epidemiology and Infection 2014; 143: 2018-2042.

5. Halliday J, et al. Bringing together emerging and endemic zoonoses surveillance: shared challenges and a common solution. Philosophical Transactions of the Royal Society of London, Series B, Biological Sciences 2012; 367: 2872-2880.

6. Freifeld CC, et al. Participatory epidemiology: use of mobile phones for community-based health reporting. PLoS Medicine 2010; 7: e1000376.

7. Vourc'h G, et al. Detecting emerging diseases in farm animals through clinical observations. Emerging Infectious Diseases 2006; 12: 204-210.

8. Robertson C, et al. Mobile phone-based infectious disease surveillance system, Sri Lanka. Emerging Infectious Diseases 2010; 16: 1524-1531.

9. Sawford K, Vollman AR, Stephen C. A focused ethnographic study of Alberta cattle veterinarians' decision making about diagnostic laboratory submissions and perceptions of surveillance programs. PLoS ONE 2013; 8: e64811.

10. Sawford K, Vollman AR, Stephen C. A focused ethnographic study of Sri Lankan government field veterinarians' decision making about diagnostic laboratory submissions and perceptions of surveillance. PLoS ONE 2012; 7: e48035.

11. Robinson PA, Epperson WB. Farm animal practitioners' views on their use and expectations of veterinary diagnostic laboratories. Veterinary Record 2013; 172: 503.

12. Wohlfender FD, et al. A review of twenty years of equine infectious disease monitoring in Switzerland: past, present and future. Journal of Equine Veterinary Science 2012; 32: S92.

13. Wohlfender F, et al. Equine infectious disease monitoring in Switzerland: past, present and future [in German]. ALP Science 2013; 543: 22-23.

14. Anonymous. Re-launch of Equinella: sentinel veterinarians wanted! [in German]. Schweizer Archiv für Tierheilkunde 2013; 155: 594-595.

15. Hauser R, Meier HP. Equinella - the monitoring of infectious equine diseases in Switzerland. In Proceedings of the 9th Symposium of the International Society for Veterinary Epidemiology and Economics, Breckenridge, Colorado, USA. Respiratory disease in horses session, p. 300, August 2000. International Symposia on Veterinary Epidemiology and Economics.

16. Struchen R, et al. Investigating the potential of reported cattle mortality data in Switzerland for syndromic surveillance. Preventive Veterinary Medicine 2015; 121: 1-7.

17. Perrin JB, et al. Assessment of the utility of routinely collected cattle census and disposal data for syndromic 
surveillance. Preventive Veterinary Medicine 2012; 105: 244-252.

18. Smyth GB, Dagley K, Tainsh J. Insights into the economic consequences of the 2007 equine influenza outbreak in Australia. Australian Veterinary Journal 2011; 89 (Suppl. 1): 151-158.
19. Waller AS. Strangles: taking steps towards eradication. Veterinary Microbiology 2013; 167: 50-60.

20. Leblond A, Hendrikx P, Sabatier P. West Nile Virus outbreak detection using syndromic monitoring in horses. Vector-Borne and Zoonotic Diseases 2007; 7: 403-410. 